\title{
CHEMICAL EVOLUTION OF DENSE CLOUDS
}

\author{
E. W. CHA PELLE, B. D. DONN, W. A. PAYNE, JR., and L. J. STIEF \\ Astrochemistry Branch, Laboratory for Extraterrestrial Physics, \\ NASA/Goddard Space Flight Center, Greenbelt, Md. 20771, U.S.A.
}

\begin{abstract}
Thermal reactions largely determine the chemical evolution of interstellar molecules during various phases associated with the evolution of interstellar clouds. A similar mechanism may be the source of the array of observed polyatomic molecules. If astronomical time scales are comparable to or less than chemical time scales, thermodynamic equilibrium will not be attained. Other factors also affect the validity of thermodynamic calculations of molecular composition. An experimental investigation of thermal reactions in a simplified system is under way. Preliminary results of the $\mathrm{HCN}-\mathrm{H}_{2}$ reaction at $1000 \mathrm{~K}$ indicate reaction times of several hours. These have been extrapolated to lower temperatures and fitted to models of the primordial solar nebula, proto stars and red giants. In these situations equilibrium would generally not be attained and reaction products would freeze out at temperatures near $700 \mathrm{~K}$ or greater.
\end{abstract}

\section{Astronomical Problem}

The origin and subsequent evolution of the complex array of molecules found in dense, heavily obscured clouds present a variety of significant astronomical and chemical problems. Several investigations, e.g., the Liege Symposium, Stellar Evolution Before the Main Sequence, (1970); Kaplan and Pikelner, (1970); and recent papers by Cameron $(1969,1972)$ have indicated that in many cases such clouds are gravitationally unstable and undergoing collapse. This process is the accepted view of the formation of young star clusters and planetary systems. During the collapse phase high temperatures and pressures occur, densities from $10^{10}-10^{16} \mathrm{~cm}^{-3}$ and temperatures to $2500 \mathrm{~K}$ appear likely. As the infall of matter ceases, the cloud temperature falls and the cloud becomes much colder again. It is during this latter stage that planet accumulation would primarily take place (Larimer, 1970; Anders, 1971).

This paper considers chemical processes that could determine the molecular composition of the cloud during the several stages of its evolution. In particular we examine thermal reactions at the relatively high interstellar densities expected during the process.

Three distinct circumstances occur to which our analysis applies.

\subsection{THE ORIGIN OF THE OBSERVED MOLECULES}

(A) If the molecules preceded collapse and star formation, the molecule formation mechanism is very uncertain but thermal processes are unlikely. However, there is a tendency for many of the observed polyatomic molecules in Orion to be concentrated in small regions associated with the infra-red source (Snyder and Buhl, 1971; Penzias et al., 1971; Barrett et al., 1971). In a paper at this symposium, Martin and Downes (1972) presented evidence for several regions of star formation in Sag B2. These are 
associated with dense, high temperature ionized regions which act as continuum sources.

(B) A possible source of the molecules in these complex cloud arrays is in expanding atmospheres of newly formed stars with subsequent ejection into the cloud. A combination of expansion and turbulence set up by the onset of radiation of hot OB stars may well be a mechanism to spread the molecule through a large volume of the surrounding cloud. Shimizu (1973) and in this symposium has considered the atmospheres of newly formed stars as molecular sources and attempted to calculate the composition. In this hypothesis thermal reactions would be a major source of molecules. The question arises as to the molecular composition when the temperature and density become so low that reactions effectively cease.

\subsection{Molecular eVolution DURing COllapse}

According to hypothesis $1.1 \mathrm{~B}$, few (if any) polyatomic molecules would be present in the cloud during collapse. However, during the subsequent cooling stage, as temperatures drop from about $2000 \mathrm{~K}$ and densities are high, thermal reactions must occur. The cooled nebula would have a complex composition. Several detailed equilibrium calculations have been made for the primordial solar nebula (e.g. Urey, 1952; Lord, 1965; Larimer, 1967; Lewis, 1972). Closely related to this process is the evolution of precollapse molecules in hypothesis 1.1A. In this situation a complex array of molecules reacts thermally during the heating phase. At temperatures of $2000 \mathrm{~K}$ or above and densities of $10^{-3}$ to 1 atm equilibrium would be quickly established. As the cloud cooled, the composition would continuously change. We inquire as to the composition to be expected when the temperature has fallen to the value occurring when, in the primordial solar nebula, the various condensation and accumulation processes occurred (Anders, 1971). In particular, we consider the validity of thermodynamic equilibrium calculations of molecular composition.

\subsection{THE ROLE OF ORGANIC MOLECULES IN PLANETARY CHEMICAL EVOLUTION}

Although this aspect of molecular phenomena is somewhat removed from the main argument of the conference, non-thermodynamic equilibrium effects may be significant and some consideration will be given to it. Pre-collapse molecules may not survive the high temperature stage in their initial array but a different array should appear during the cooling phase as outlined in 1.2. Pre-collapse molecules may not exist but, as in hypothesis $1.1 \mathrm{~B}$, they arise in the primordial stellar nebula following star formation. Under any circumstance it thus appears that an array of organic compounds is present when planets accumulate around newly formed stars. What happens when massive, planetary objects form? At the final stage of accumulation the solid material will have accreted into a hierarchy of planetesimals (Savronov, 1964, 1966). These would impact on cool planetary surfaces causing local high temperatures but with rapid cooling. Organic compounds may tend to be destroyed as Sagan (1965) suggested but as in hypothesis 1.2, the composition of the vaporized constituents after cooling may well contain a disequilibrium excess of organic molecules. Oro's (1965) proposal 
for the initiation of biochemical evolution may have considerable merit according to this analysis.

As a model for the astronomical aspects of the collapse we use Cameron's (1972) recent analysis of the formation of the solar system. A massive cloud collapses and fragments into units containing up to ten solar masses. The Sun and planets form from a two solar mass fragment. Within $1 \mathrm{AU}$ of the Sun the temperature reaches about $2000 \mathrm{~K}$ and cools with a time scale of ten years. At $10 \mathrm{AU}$ the temperature is much less than a thousand degrees with a cooling time of 100-1000 yr.

\section{Chemical Problems}

\subsection{THEORY}

Molecular equilibrium calculations for cool stellar atmospheres, interstellar clouds, and primordial stellar and planetary nebula have assumed thermodynamic equilibrium. For several reasons the applicability of thermodynamic equilibrium to these objects is open to question. (1) Variations in temperature and pressure will invalidate such analyses if the time scale for attaining equilibrium is of the order of, or less than the time scale for astronomical changes. A determination of each time scale must be made. (2) Equilibrium calculations only yield results for those species that are initially included in the program. It does not predict species. Unexpected or non-equilibrium species regardless of concentration would not appear in the results nor would their absence cause any obvious effects.

In order to investigate the question of the rate of approach to equilibrium and the ultimate molecular composition we have begun a series of experiments at high temperatures. Because of the continuously changing temperature and pressure, equilibrium will not be attained in most cases except perhaps at the highest temperatures. An extremely complex set of reactions would determine the composition at each stage. As the temperature decreases during the cooling phase, each reaction and molecular species will behave differently. Chemical processes will be frozen out and metastable products will tend to be preserved. Because of these problems with a theoretical calculation, this experimental study was begun. This procedure develops its own set of difficulties which must be overcome. Experimental techniques are being developed to accomplish this.

The first series of experiments are designed to determine the reaction rate as time scale for approach to equilibrium and its temperature dependence. The measurement of an overall activation energy will permit extrapolation of the reaction rates to lower temperatures where rates are too slow for experimental determination.

\subsection{EXPERIMENTS}

Mixtures of $\mathrm{HCN}$ and $\mathrm{H}_{2}$ in ratios of $1: 100,1: 000,1: 3000$ were prepared and heated in quartz reaction vessels at $1093 \mathrm{~K}$ for various times. The starting pressure at room temperature was approximately 300 torr. At the operating temperature, the pressure in the vessel was about $1.3 \mathrm{~atm}$. As the final composition did not noticeably depend 
upon the $\mathrm{HCN}: \mathrm{H}_{2}$ ratio, the experiments were run at the 1:100 ratio to enhance product yield and improve the accuracy of the results. After the nominal duration of heating the furnace was turned off and allowed to cool. The gradual cooling process had a significant effect on the shortest $(2 \mathrm{~h})$ heating time only.

The fraction of $\mathrm{HCN}$ remaining is a measure of the completion of the reaction. Equilibrium calculations (Tsuji, 1964) show that at equilibrium the residual HCN concentration would be $0.1 \%$ at $1090 \mathrm{~K}$ and $0.06 \%$ at $1170 \mathrm{~K}$. Figure 1 shows the residual $\mathrm{HCN}$ percentage as a function of heating time. From Figure 1, we obtain a half life $\left(e^{-1}\right)$ of $18 \mathrm{~h}$. $\mathrm{HCN}: \mathrm{H}_{2}=1: 100$ mixtures were also reacted at $1170 \mathrm{~K}$ and $980 \mathrm{~K}$. The decay times are listed in Table I for the three temperatures. The effective activation energy for the reaction, obtained from the dependence of reaction rate on temperature, is $20-25 \mathrm{kcal}^{\mathrm{mole}}{ }^{-1}$. A value this low is unexpected as activation energies for reactions of stable molecules of this type generally fall between $40-100 \mathrm{kcal}^{\mathrm{mole}} \mathrm{e}^{-1}$ (Bahn, 1968; Kondratiev, 1972).

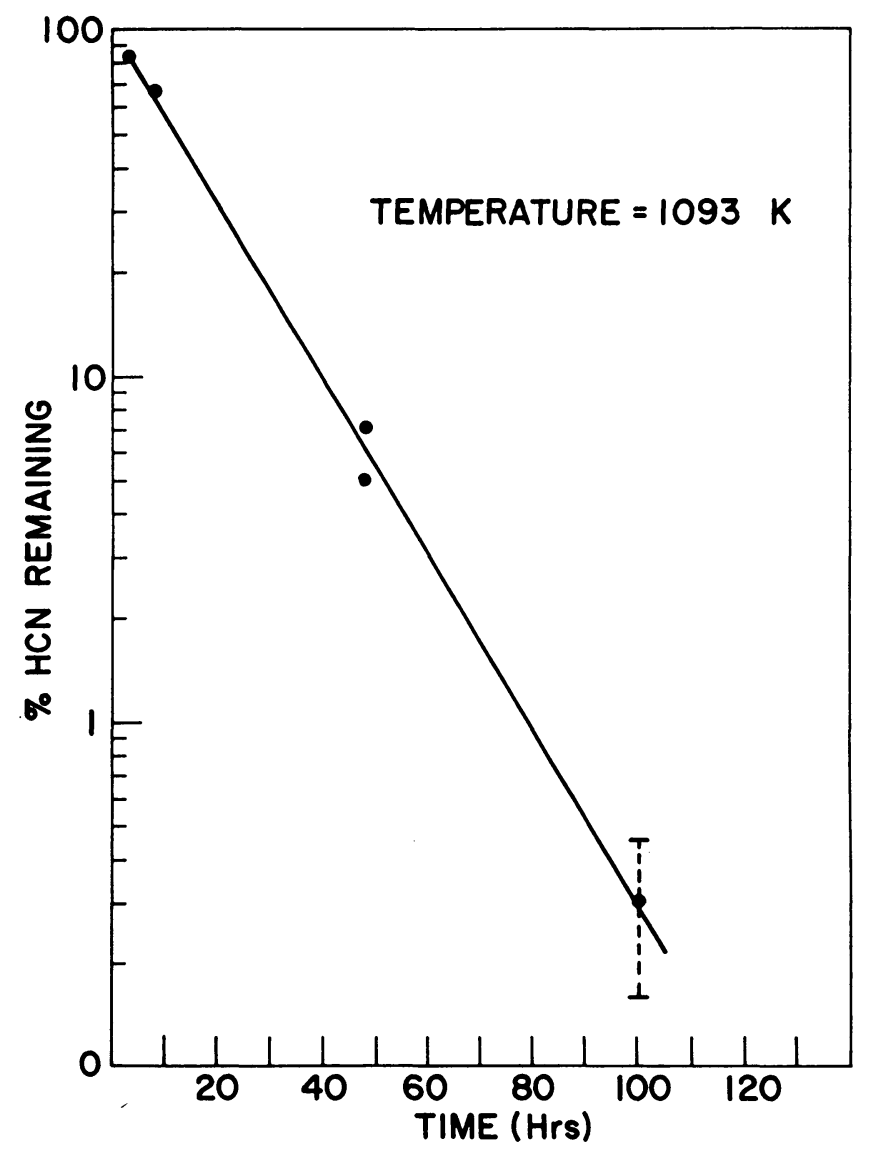

Fig. 1. Dependence of $\mathrm{HCN}-\mathrm{H}_{2}$ reaction on time. The dashed vertical bar at $100 \mathrm{~h}$ is the uncertainty of measuring that quantity of $\mathrm{HCN}$ on the mass spectrometer. 
A few runs were made with an oxygen or water concentration equal to that of hydrogen cyanide in the mixture. The essential result, the $\mathrm{HCN}$ decay time, was similar to the previous case.

There is a possibility that surface reactions made a significant contribution to the observed rate. A quartz vessel was filled with 20 quartz tubes, thereby increasing the surface to volume ratio by a factor of 9 . In $10.5 \mathrm{~h}$, the $\mathrm{HCN}: \mathrm{H}_{2}$ mixture at $1090 \mathrm{~K}$ showed a $2 \% \mathrm{HCN}$ residue whereas from Figure 1, the anticipated residue was $55 \%$. This indicates a major surface reaction contribution which would explain the low activation energy derived from the experiment. It also means that the $18 \mathrm{~h}$ half-life is a lower limit.

An additional factor affecting the experiment is contamination by gases driven from the walls of the reaction vessel at the temperatures of $1000 \mathrm{~K}$ over several hours. Significant hydrogen loss via diffusion through the walls occurs as well. For example, in forty eight hours $20 \%$ of the hydrogen was lost.

Work on these high temperature reactions related to molecular equilibrium in astrophysical systems is continuing in order to refine the experiments and extend the results to more systems and conditions.

\subsection{APPliCATION to CLOUd EQUILIBRIUM}

We return to the question of the approach to thermodynamic equilibrium in a collapsing or cooling cloud or in an expanding stellar atmosphere or shell. Hydrogen cyanide is one of the more abundant interstellar molecules and also is an important reactive precursor in biochemical evolution (Calvin, 1969). Consequently the behavior of this compound not only gives some indication of the approach to equilibrium in a cloud but also yields information on the ultimate concentration of an important prebiotic specie.

The dependence of the rate of the $\mathrm{HCN}-\mathrm{H}_{2}$ reaction on surface to volume ratio indicates that the experimental half-life of $18 \mathrm{~h}$ at $1090 \mathrm{~K}$ is a lower limit. In Table I we use this value and extrapolate to lower teperatures using activation energies of 20 and $50 \mathrm{kcal} \mathrm{mole}^{-1}$. The higher value yields results that may be more applicable in the absence of extensive surface reaction. Further experiments will seek to establish the gas phase activation energy.

Table II shows that if the effective activation energy were as low as $20 \mathrm{kcal} \mathrm{mole}^{-1}$, equilibrium would take approximately $300 \mathrm{yr}$ at $500 \mathrm{~K}$. In Cameron's latest model of the solar nebula this temperature is reached between 5 and 10 AU. His calculated life-

TABLE I

Half life of $\mathrm{HCN}-\mathrm{H}_{2}$ reaction (measured)

\begin{tabular}{rc}
\hline$T(\mathrm{~K})$ & $\tau\left(e^{-1}\right)(\mathrm{h})$ \\
\hline 1170 & 9 \\
1093 & 18 \\
980 & 53 \\
\hline
\end{tabular}


times are comparable to the chemical lifetime raising a question of the approach to thermodynamic equilibrium. Half lives calculated using an activation energy of 50 $\mathrm{kcal}_{\mathrm{mole}}^{-1}$ suggest that there would only be sufficient time to approach equilibrium within about $1 \mathrm{AU}$.

TABLE II

Half life of $\mathrm{HCN}-\mathrm{H}_{2}$ reaction (theory)

\begin{tabular}{lll}
\hline & \multicolumn{3}{l}{ Activation Energy } \\
\cline { 2 - 3 } $\begin{array}{l}E-20 \\
(\mathrm{Kcal} \mathrm{mole})\end{array}$ & $\begin{array}{l}E-50 \\
\left(\mathrm{kcal} \mathrm{mole}^{-1}\right)\end{array}$ \\
\hline 2000 & $0.27 \mathrm{~h}$ & $2 \mathrm{~s}$ \\
1500 & $1.48 \mathrm{~h}$ & $140 \mathrm{~s}$ \\
1000 & $40 \mathrm{~h}$ & $147 \mathrm{~h}$ \\
900 & $120 \mathrm{~h}$ & 101 days \\
800 & $20 \mathrm{day}$ & $94 \mathrm{yr}$ \\
700 & $123 \mathrm{day}$ & $7.2 \times 10^{3} \mathrm{yr}$ \\
500 & $270 \mathrm{yr}$ & $1 \times 10^{9} \mathrm{yr}$ \\
\hline
\end{tabular}

In expanding atmospheres of red giant stars or in proto-stars the astronomical time scale is days or months. The present analysis indicates temperatures close to $1000 \mathrm{~K}$ would be required to attain a close approach to equilibrium.

Reactions caused by impact of planetesimals on the earth's surface near the end of its accumulation are very complex to analyze. The rapid change in temperature and pressure in the vapor suggest an extremely non-equilibrium final composition. The present experiments give some support to the idea that organic compounds may have been present on the surface of the newly-formed Earth.

There is some observational evidence that suggest organic compounds were present when objects accumulated in the solar nebula. A number of carbon radicals, $\mathrm{CH}, \mathrm{CN}$, $\mathrm{C}_{2}$ and $\mathrm{C}_{3}$, occur in cometary atmospheres. More complex parent compounds were almost certainly present in the primordial nebula from which comets accreted. If not originally present in the inner region, molecules like $\mathrm{HCN}$ could have formed in the non-equilibrium impact reactions.

The chemical theory and experiments are preliminary and the astronomical models are also very uncertain. Hence, no definite conclusions can be reached. The present analysis does point out that non-equilibrium processes may become very important in determining the molecular composition of cosmic clouds. This possibility must be taken into account in drawing conclusions from calculated compositions.

A continuing study of this problem is under way. We will investigate more thoroughly the limits of validity of thermodynamic equilibrium in the astronomical systems considered here. Procedures for estimating molecular compositions when thermodynamic equilibrium do not apply are also being examined. 


\section{References}

Anders, E.: 1971, Ann. Rev. Astron. Astrophys. 9, 1.

Bahn, G. S.: 1968, Reaction Rate Compilation for the $\mathrm{H}-\mathrm{O}-\mathrm{N}$ System, Gordon and Breach, N.Y.

Barrett, A. H., Schwartz, R. P., and Waters, J. W.: 1971, Astrophys. J. 168, L101.

Calvin, M.: 1968, Chemical Evolution, Oxford University Press, N.Y.

Cameron, A. G. W.: 1969, in S. S. Kuman (ed.), Low Luminosity Stars, Gordon and Breach, N.Y., p. 423.

Cameron, A. G. W.: 1972, preprint submitted to Icarus.

Kaplan, S. A. and Pikel'ner, S. B.: 1970, The Interstellar Medium, Harvard Univ. Press. Cambridge, Mass.

Kondratiev, V. N.: 1972, in R. M. Fiestrom (ed.), Rate Constants of Gas Phase Reactions-Reference Book (transl. by L. J. Holtschlag), Office Standard Reference Data, N.B.S., National Technical Information Service, Washington, D.C., U.S.A.

Larimer, J. W.: 1967, Geochim. Cosmochim. Acta 31, 1215.

Lewis, J. S.: 1972, Icarus 16, 241.

Liège Astrophysics Symposium: 1970, 'Stellar Evolution before the Main Sequence', see particularly introductory reports and references, Univ. of Liege, 1970.

Lord, H. C.: 1965, Icarus 4, 279.

Oro, J.: 1965, in S. W. Fox (ed.), The Origin of Prebiological Systems, Academic Press, N.Y., p. 137.

Penzias, A. A., Solomon, P. M., Wilson, R. W., and Jefferts, K. B.: 1971, Astrophys. J. 168, L53.

Sagan, C.: 1965, in S. W. Fox (ed.), The Origin of Prebiological Systems, see discussion following paper by Oro, p. 102, Academic Press, N.Y.

Savronov, V. S.: 1964, Techtonophysics 1, 217.

Savronov, V. S. (1966) Soviet Astron. AJ 9, 987.

Shimizu, M.: 1973, Prog. Theor. Phys. 49, 153.

Snyder, L. E. and Buhl, D.: 1971, Astrophys. J. 163, L47.

Tsuji, T.: 1964, Ann. Tokyo Astron. Obs. 9, 1.

Urey, H. C.: 1952, The Planets, Yale Univ. Press, New York. 